\title{
Microfabricated Sensors for Medical Applications
}

\author{
Ben Kloeck, Nicolaas F. de Rooij \\ Institute of Microtechnology, University of Neuchâtel, \\ 2000 Neuchâtel, Switzerland
}

\section{Introduction}

Mainly because of their size, a typical application ficld for integrated sensors is the biomedical world, and more preciscly the in-vivo determination of physical and physiological parameters. One important parameter is the pressure of body fluids. Piczoresistive pressure sensors are cheap to fabricate in mass and casy to read-out. Morcover the dimensions of piczoresistive sensors can be made very small. Therefore, the future of piezoresisitve pressure sensors is in cheap disposable catheters for invasive measurements with external power supply. The first section of this paper shows how such a pressure sensor can be applied for that purpose by mounting it in a $G F$ heart catheter.

Two more applications are presented concerning catheters that contain pressure sensor chips which are based on the technology developed at the Institute of Microtechnology. The first is an 8I: double-lument catheter for the investigation of the csophageal function. A new sensor chip was designed for this application, using a double-side integration technique specially developed for this purpose, and the back-side contact technology. It consists of a pressure sensing part and a pll sensing par. The advantages of the sensor design with back-side contacts are outlined. In-vitro and preliminary in-vivo experiments are presented, which show satisfactory results for simultancous pressure and pll measurements.

The second example is a $4 \mathrm{~F}$ muscle catheter, developed to measure pressures and forces in the muscle tissue. Ilere the special feature is the very small lateral dimension of the sensor chip.

Another area where microfabrication of sensors can be successfully used for applications in the biomedical field is that of chenical sensors.

The fabrication technology for backside contacted ISFETs, similar to that used in the combined pll-pressure sensor, will be discussed in more detail. $\Lambda$ micromachined flow cell for multi-ion sensing will be presented. The fabrication of this device is based on CMOS tcchnology, combined with anodic bonding of machined glass as well as deposition on ion-sclective polymeric membrancs.

Finally, the fabrication, characıcristics and application of a planar amperometric glucose microclectrode will be discussed.

\section{Bressure sensor for heart cathether}

Classical heart catheters are essentially tubes filled with physiological solution that is in contact with the blood through a llexible membrane in the catheter tip. The pressure is measured extemally at the other end of the catheter. This system works well for static pressures, but fast pressure changes are damped by the pliancy of the catheter walls. Evidently, this problem docs nut occur if the pressure is measured and transformed to an clectrical signal, direclly in the catheter tip [1-5].

The pressure sensor presented in this work was mounted in a $6 F$ catheter ( $2 \mathrm{~mm}$ outer diameter) by Keller $\Lambda \mathrm{G}$ für Druckmesstcchnik (Winternhur). The catheter tip was made of stainless stecl and the tube of polyurethane. The piczoresistors are tumed toward the inside of the catheter, and only the membrance cavity is cxposed to the external environment.

The catheter has been tested in-vitro, dry and in plysiological salt solutions. The stability was proven to be satisfactory: less than 1 mbar drift in $\mathbf{4 2}$ hours. So far the catheter has not bect used for invivo experiments.

\section{Dll-pressure sensor for stomach calhcle}

\subsection{Introduction}

At present, esophageal catheters only allow the measurement of cither pll (acidity) or pressure scparately. For some studics and diagnoses however, the measurement of both simultaneousiy is felt as a need to obtain a more complete image of the pysiological phenomena involved [6-8]. Onc important application is the investigation of the lower csophageal sphincter (the ring-shaped muscle that by contraction closes the access to the stomach), and in particular the reflux of stomach acid into the esophagus that occurs if this muscle is malfunctioning. Diagnosis is complicated by the fact that the symptoms are often intermittent and are triggered by stimuli like stress or temparature extremes. Another interesting application for medical and pharmacological rescarch is the dynamic and chemical activity of certain medicines and drugs. The advantage of having a pressure sensor at the same time is that also informations about the movement of the esophageal tract are obtained.

Today's commercially available csophagcal catheters for $\mathrm{pll}$ monitoring use cither antimony or glass clectrodes. Antimony clectrodes have the advantages that they are sinall and very cheap, and thus suited for disposable use. On the other hand, however, they are incxact and suffer from agcing and hysteresis, since their electrode reaction is essentially a corrosion process. Morcover, at low pll valucs there is an accumulation of reaction products around the clectrode, which causes false pH readings. Glass electrodes are exact to a tenth of a pll or better, and stable. The disadvantages are that they are fragile and that they have a high impedance. Still, for the moment the glass electrodes are the most reliable devices that are on the market, but if they have to be combined with pressure sensors, their size increases, causing thus a lot of discomfor for the patient who receives the catheter through his nose. Compared with these existing systems, the silicon based sensor has the advantages of small dimensions, good accuracy and stability, and above all, a vast flexibility to integrate two or more sensors on one chip and on more sites in the catheter. In this section, a silicon based pllpressure catheter (double-lumen), with the possibility for the integration of a reference clectrode in one lumen, is discussed. [9.10].

\subsection{Sconsor chip fabrication}

The sensor chips are fabricated by standard microclectremic and micromachining processes on double-side polished, 3 inch wafers (boron-doped, 3.5 $\Omega \mathrm{cm}, 380 \mu \mathrm{m}$ thick). 1 very special fcature of this chip is that components are integrated on both sides. This is because one of the major design goals was to locate all the contact pads on the back side of the chip. since it has to be mounted in a catheter. Therefore the pressure sensor is integrated on the back side and tic pll ISFLET, which must be exposed to the sulution, on the front side, but with contact feed-throughs through the chip. as is shown schematically in Fig. 1. The diunensions of the chip are 1.5 $\mathrm{m}: \mathrm{n}$ by $4.0 \mathrm{~mm}$. 


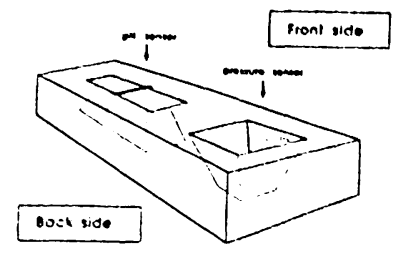

Fig. 1: Transparent scheme of the combined pH-pressure sensor

The pressure sensor consists of four boron-diffused piezoresistors at the edges of a thin membrane and connected in a Whentstone bridge configuration. The output voluge is proportional to the pressure difference across the membrane and to the applied bridge voluage. The membrane is etched in poussium bydroxide from the front side of the wafer and its thickness is controlled by a four-electrode electrochemical etch-stop. A $10 \mu \mathrm{m}$ thick epitaxial layer of n-type silicon is grown on a p-type silicon substrate. The p-type substrate is etched and etching is stopped at the epi-layer/substrate junction by applying a passivating potential to the epi-layer. The thickness of the remaining silicon membrane is thus defined exactly by the thichness of the epitaxial layer and nor influenced by the taper of the wafer.

The pH-ISFET fabrication resembles the standard fabrication process [11] with one exception, i.e. the location of the drain and, source contacts. Under the source and drain regions, holes are etched from the back side of the wafer, leaving a membrane of about $10 \mu \mathrm{m}$ thick. A high phosphorous diffusion through this membrane, covered with aluminium establishes the electrical contacts to the source and drain from the back side of the chip. The process will be discussed in more detail in section 5 .

\subsection{Catheter encapsulation}

The pH-pressure chip is mounted in a double lumen $8 \mathrm{~F}$ catheter $(2.7$ $\mathrm{mm}$ diameter). Fig. 2 shows the important advantage of the backside contacts: this approach allows the sensitive part to be almost at the same level as the outside surface of the catheter, avoiding thus the presence of a cavity in the tip, where local $\mathrm{pH}$ could differ from the effective $\mathrm{pH}$ in the stomach. The gate of the $\mathrm{pH}$ sensor and the back side of the pressure membrane are directly in contact with the solution. The catheter contains seven wires to contact the pressure sensor, two for the pH ISFET and one for possible integration of an $\mathrm{Ag} / \mathrm{AgCl}$ reference electrode in the second lumen. The chips were mounted in the catheter by Keller AG für Druckmesstechnik (Winterthur).

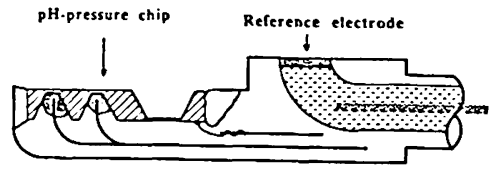

Fig. 2: Cross-section of the catheter tip, showing the chip encapsulation

\subsection{Measurement set-up}

For the in-vitso measurements the catheter was inserted in a vertical flexible tube that was consecutively filled with different $\mathrm{pH}$ buffer solutions. The upper liquid level was varied to yield pressure variations in the order of 100 mbar. Thus pressure and $\mathrm{pH}$ could be measured simultaneously. Merck pH buffer solutions were used. The $\mathrm{pH}$ was measured with an in-house built ISFET amplifier: a feedback control system that applies a constant $V_{D S}$, measures $I_{D S}$ and adjusts $\mathrm{V}_{\mathrm{GS}}$ through an $\mathrm{Ag} / \mathrm{AgCl}$ reference electrode, in order to keep this $I_{D S}$ constant. $V_{G S}$ is then proportional to the $\mathrm{pH}$ of the solution. The pressure was measured with a Wheatstone bridge amplifier supplied by Keller AG.

For the in-vivo measurements only batuery powered equipment was used. A portable in-house built ISFET amplifier with skin reference electrode, Keller DPM 80 Wheatstone bridge amplifier and MIC GastrograpHs to obtain digital recondings of the measurements. The senson were routinely calibrated before use.

\subsection{Resuls and discussion}

In-vitro measurements have shown that the pressure sensor had good linearity and a sensitivity of $8 \mathrm{mV} / \mathrm{Vbar}$. The $\mathrm{pH}$ sensor had a sensitivity of $56 \mathrm{mV} / \mathrm{pH}$ and stability better then $0.2 \mathrm{mV} / \mathrm{h}$. Preliminary in-vivo messurements were carried out at the Insel Spital in Bem, in the stomach of normal volunteer. A typical simulaneous recording of pressure and $\mathrm{pH}$ is shown in Fig. 3. At the same time a glass pH-electrode (Ingold) was inserted in the stomach and the readings were observed to be parallel, with only slight differences between both electrodes caused by their different position in the stomach. One of the advantages of the pressure recording is that the moment when the catheter passes through the sphincter can be determined exactly.

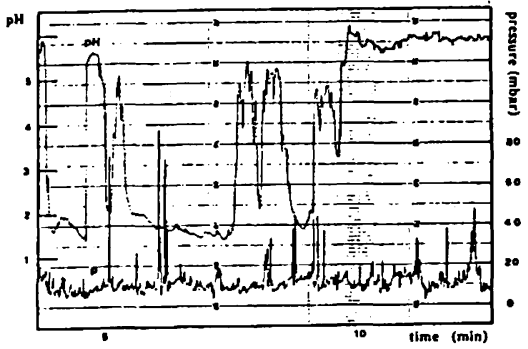

Fig. 3: Simultaneous recording of pressure and $\mathrm{pH}$ in the stomach of a normal volunteer

\subsection{Conclusion}

The in-vitro characteristics of the $\mathrm{pH}$-pressure sensor are comparable to equivalent single transducers. The integration of $\mathrm{pH}$ and pressure sensors on one chip with back-side contacts enables an easy catheter mounting and adequate encapsulation. To obtain better spatial information, more measuring sites in the catheter are feasible. The integration of a reference electrode has been tested in vitro. The first in-vivo tests with skin electrodes showed a good response for both $\mathrm{pH}$ and pressure recordings.

\section{Eorce sensor for muscle catheter}

\subsection{Introduction}

The activitiy of muscles can be investigated by inserting athin catheter longitudinally into the muscle bundle. $\dot{A}$ force sensor in the side-wall of the catheter gives information on the lateral action of the muscle. A sensor chip was designed to replace an older system where minuscule strain gauges were glued on a beam. Its fabrication involved individual assembling of each sensor and laborious handling of the microscopic parts. Evidently this application is a typical challenge for integrated sensor technology. This section presents a first generation of this type of sensors, to show the feasibility of fabricating silicon integrated force sensors with very small lateral dimensions.

\subsection{Chip fabrication}

The chip had to be designed to be mounted in a $4 \mathrm{~F}$ catheter $(1.3 \mathrm{~mm}$ outer diameter). Its dimensions were limited at maxiumum $0.6 \mathrm{~mm}$ witdth and $6 \mathrm{~mm}$ length. A mesa was required on one end of the chip, to touch the thin membrane in the catheter opening, as shown schematically in Fig. 4. The chip thickness was $0.2 \mathrm{~mm}$, and the total thickness of the chip and the mesa was $0.4 \mathrm{~mm}$. The operational range was $40 \mathrm{mN}$ and the maximum allowed deflection of the beam $0.05 \mathrm{~mm}$. If the beam deflects too much, then the muscle tissue presses on the border of the catheter opening, and not on the mesa anymore. To increase the force sensitivity, a cavity was etched 
under the resistors. It is easily calculated that the sensitivity is proportional to the inverse of the equare of the remaining thickness. The only restriction is the deflection at maximum force. It was calculated that a thickness of $100 \mu \mathrm{m}$ would give a beam deflection at $40 \mathrm{mN}$ of less than $50 \mu \mathrm{m}$.

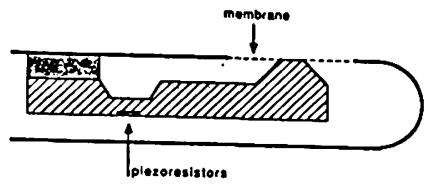

Fig. 4: Cross-section of the force sensor and the catheter encapsulation

The sensors were fabricated on three-inch phosphorous doped silicon wafers of $(100)$ orientation. The nominal thickness was 381 $\mu \mathrm{m}$ and the resistivity $1-10 \Omega \mathrm{cm}$. The fabrication sequence was similar to that of the pressure sensors, with the exception of the doping concentration of the piezoresistors. Since no Wheatstone bridge configuration was used for these sensors, the doping concentration was chosen high, $2 \times 10^{20} \mathrm{~cm}^{-3}$ at the surface, in order to reduce the temperature dependence of the piezoresistance coefficients. The sheet resistivity was $20 \Omega$ per square. After fabrication of the piezoresistors, the wafers were etched in $40 \%$ potassium hydroxide at $60^{\circ} \mathrm{C}$, to obtain the chip profile that is illustrated in Fig. 4. The etching was done in two steps: first the aforementioned cavity was etched, and then the oxide mask was removed, except on the mesas, to etch the chip to the required thickness of $200 \mu \mathrm{m}$. Finally the wafes were diced into individual sensors. Fig. 5 shows the design of two sensor types that were realized; one was $0.6 \mathrm{~mm}$ wide and the second only $0.3 \mathrm{~mm}$.

\subsection{Measurementset-up}

For in-vitro measurement, the sensors were glued on a PCB in a way that allowed vertical deflection of the bearn. A special measurement set-up was developed to measure simultaneously the force applied on the mesa, the deflection of the beam and the resistor change. For this purpose, an I-DIM displacement sensor (Cary Compar, Le Locle) was mounted vertically on a support. In the sensor head, an inductive stylus was suspended on two horizontal spring blades with a precisely known spring constant. The I-DIM head could move vertically, and a second displacement sensor allowed to measure its movement. When the stylus just touched the beam, the applied force was zero and the reading of the displacement of the stylus (caused by its own weigth) was set to zero. When the I-DIM head was moved down, the applied force and the stylus displacement were read from the display. The deflection of the beam was the difference of the readings of the I-DIM and the second displacement sensor. In this way increasing forces were applied and the corresponding resistance changes and deflections were measured.

For the temperature characterization, the PCB-mounted force sensors were placed in a fumace. The sensitivity was measured by a two-point calibration, using a known weight.
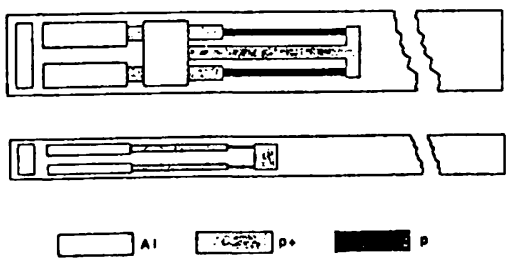

Fig. 5: Two designs of the force sensor: one with a chip width of $0.6 \mathrm{~mm}$ (upper), and one of 0.3 (lower)
This paragraph summarizes the results of the measurements on four sensors. No functional differences were observed between the two sensor types $(0.6 \mathrm{~mm}$ and $0.3 \mathrm{~mm})$. Fig. 6 shows s typical recording of the resistance change and the deflection of the bearn as a function of applied force. The sensitivity was $0.5 \Omega / \mathrm{k} \Omega \mathrm{mN}$. For a range of $40 \mathrm{mN}$, this results in a full scale resistance change of $20 \Omega / \mathrm{k} \Omega$. The deflection at $40 \mathrm{mN}$ was $34 \mu \mathrm{m}$, which is within the required specification limit of $50 \mu \mathrm{m}$. It is noted that the sensitivity could be increased by taking a lower doping concentration for the resistors. For the used concentration the longitudinal piezoresistance coefficient in the $\langle 110\rangle$ direction was $30 \times 10^{-11} \mathrm{~Pa}^{-1}$, as opposed to e.g. $50 \times 10^{-11} \mathrm{~Pa}^{-1}$ for doping concentration of $10^{-19} \mathrm{~cm}^{-3}$. However, that would be at the expense of a higher temperature coefficient. In the interval from room termperature to $70^{\circ} \mathrm{C}$, the temperature coefficient of the resistor (TCR) was measured to be $1400 \mathrm{ppm} /{ }^{\circ} \mathrm{C}$ and of the sensitivity (TCS) $1700 \mathrm{ppm} / \mathrm{C}$.

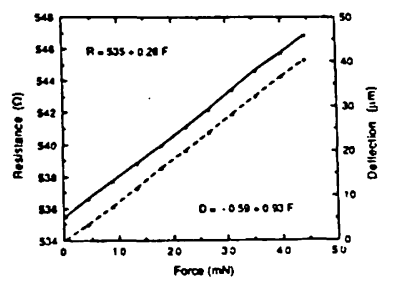

Fig. 6: Resistance change (-) and beam deflection (---) as a function of applied force.

\subsection{Conclusion}

The chip presented in this section was mainly a feasibility study as a first step towards a more sophisticated device. In particular temperature compensation was completely lacking on this chip. Because of the small dimensions (especially for the $0.3 \mathrm{~mm}$ chip), it is hardly possible to integrate two supplementary transverse piezoresistors and build a Wheatstone bridge. A full bridge would require the two transverse resistors to be placed on one line and besides the longitudinal ones, in order to sense the same mechanical tensions.

A valuable solution of this problem that requires only longitudinal resistors and still includes temperature compensation is propused here. If two identical pairs of longitudinal resistors are integrated on both sides of the chip, as schematically shown in Fig. ?. Then deflection of the chip causes the two resistors on one side to increase and the two on the other side to decrease. As for the pressure sensors, the four resistors can be connected in a Wheatstone bridge configuration, and temperature changes will in first order be a common mode effect and will not appear at the differential output of the bridge. Using the double-side integration and back-side contact technologies which were developed for the $\mathrm{pH}$-pressure chip, such a force sensor can easily be fabricated with very small lateral dimensions. To decrease the size of the contaca feed-throughs, very thin wafers should be used (100 to $150 \mu \mathrm{m}$ thick).

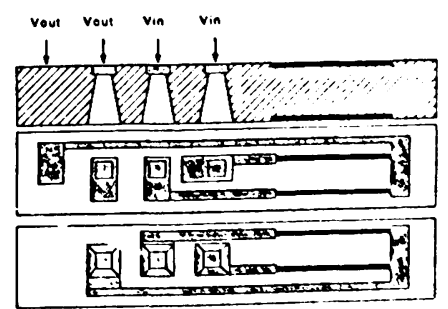

D P. $p$

Fig. 7: Design proposition for a very narrow force sensur, using double-side integration and hack-side comlact technologies (the figure dies nox show the Al amiact pads). 


\section{Slicrofabricatad chemicaleenson}

\subsection{Dll-ISEDS}

Ion-concentration or ion-activity sensors usine field effoct transistors are known since almont iwenty years and denoted as lonsensitive Field Effect Transistors or ISFETs. Despite their promising perspectives, a number of problems related to encapsulation and drift are still not solved and hamper a wide spread use. In order to improve the encapsulation we developed PH-ISFETs with back-side contacts. [12]

To fabricate feed throughs in a silicon wafer, several techniques can te utilized. A nther simple method which can be used on wafer level, is the anisotropic etching of the silicon substrate with e.g. porassium hydroxide to form holes in the substrate leaving a thin membrane the thickness of which can be controlled. Electrical contacts to the source and drain regions on the topside of the chip. can be made by diffusion of drain/source dopant through this thin silicon membrane. On \& double side polished wafer (p-type $3.5 \Omega \mathrm{cm}$, thickness $380 \mu \mathrm{m}$ ) an oxide layer of about $1.5 \mu \mathrm{m}$ is grown as masking layer for the potassium hydroxide etching. Then the openings for the drain and source back-side contacts are made. The holes in the silicon are etched in a $40 \%$ potassium hydroxide solution at $60^{\circ} \mathrm{C}$. The wafer is etched until less than $10 \mu \mathrm{m}$ of silicon is left leaving a diaphragm of about $80 \mu \mathrm{m} \times 80 \mu \mathrm{m}$ at the bottom
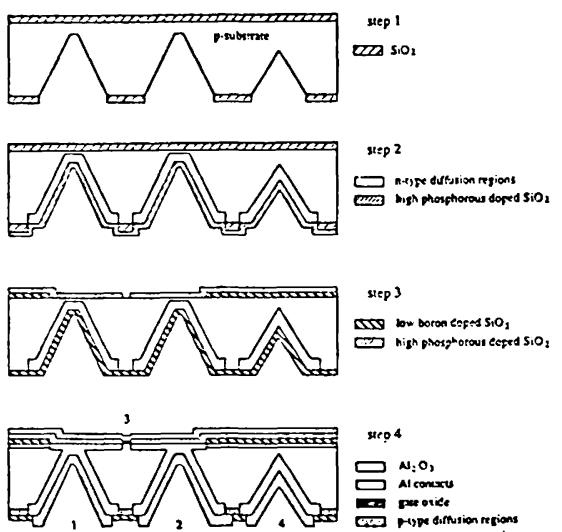

Fig. 8: Fabrication sequence of back-side pH-ISFETs

The back-side openings are now enlarged to enable the deposition of a phosphorous doped silicon oxide layer by chemical vapour deposition (CVD). After the diffusion of the phosphorous into the silicon all oxide layers are etched away and a boron doped silicon oxide layer is deposited on both sides of the wafer. Then the drain and source areas are opened and a phosphorous doped silicon oxide layer is grown (typically $900 \AA$ ) and at the same time the drain and source areas are formed together with the channel stop at both sides of the wafer. Alumina is then deposited by CVD as $\mathrm{pH}$-sensitive layer $(600 \AA)$. After opening the contact windows on the back-side, aluminium contacts are formed by lift-off and the source and the substrate are directly connected. Furthermore some MOSFETs are made for on wafer testing. A diagram of the sensor fabrication is shown in Fig. 8.

The characteristics of the $\mathrm{pH}$ sensor are the following: drift less then $0.5 \mathrm{mV} / \mathrm{h}$ and $\mathrm{pH}$ sensitivity of about $56 \mathrm{mV} / \mathrm{pH}$.

A carbon dioxide electrode based on the Severinghaus principle has been developed using the back-side contacted pH-ISFETs (Fig. 9). The $\mathrm{pH}$-ISFET is used as the internal $\mathrm{pH}$-sensing element and a temperature sensitive diode is integrated on the same chip [13].

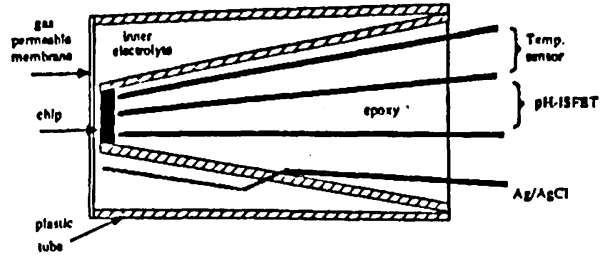

Fig. 9: Schematic diagnm of the $\mathrm{CO}_{2}$-sensor

\subsection{Multi-ion sensors}

In order to realize FET-based sensors sensitive to other ions than $\mathrm{H}^{+}$-ions, polymeric membranes with ionophores can be applied. However, the fixation of this type of membranes to the basic sensor structure is a problem. Glass encapsulations based on anodic bonding can solve both the problem of membrane fixation and packaging. The types of glass commonly used for this process are known to be highly resistant against chemical attack. Prior to the bonding process, holes are drilled through the glass in order to have access to the sensing area as well as to the electrical contacts. The glass hole provides excellent physical fixation of the membrane. Additional treatment of the glass before membrane deposition can further improve the adhesion of the membrane to the sensor [14].

The basic design of our structure is shown schematically in Fig. 10. It consists of a silicon substrate into which a field effece transistor has been realized. The gate of the transistor consists of a silver thin film, using a titanium adhesion layer. On top of the silicon substrate, the glass plate is applied by anodic bonding on an intermediate polysilicon layer. After the anodic bonding procedure. the silver layer (gate) is partially converted into a silverchloride layer in order to provide a stable electrochemical interface with the covering mernbrane.

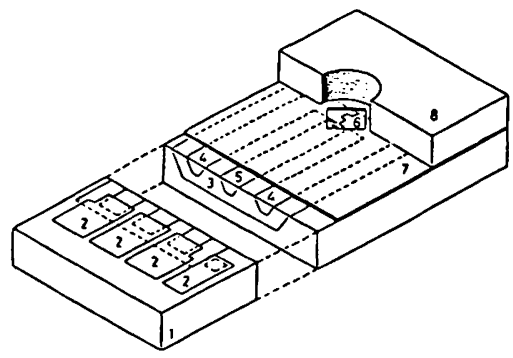

Fig. 10: Diagram of the glass -encapsulated pH-sensitive ISFET 1) n-type substrate, 2) contact pads, 3) p-well, 4) drain and source (ntype), 5) conductor (n-type) (forms diode with well) with at the end a PUAl electrode (black triangle connected during the anodic bonding with a metal layer to the gate), 6) gate area, exposed to the solution after the anodic bonding, 7) polysilicon layer, 8) glass (only pardy shown but covers completely the polysilicon layer

The sensitivity of these sensors to chloride ions was $58.5 \pm 1$ $\mathrm{mV} / \mathrm{pCl}$ at $25^{\circ} \mathrm{C}$. The observed drift was less then $10 \mu \mathrm{V}$ per hour at $25^{\circ} \mathrm{C}$ in $\approx 0.1 \mathrm{M} \mathrm{KCl}$ solution. The $\mathrm{pH}$ characteristics of a glass encapsulated ISFET without the $\mathrm{Ag} / \mathrm{AgCl}$ gate is comparable to those mentioned above.

A flow-through cell based on the above described technology has been realized. It allows simultaneous measurement of $\mathrm{H}^{+}, \mathrm{K}^{+}$and $\mathrm{Ca}^{++}$-ion activities [15] For the $\mathrm{K}^{+}$-ion activity and $\mathrm{Ca}^{++}$-ion activity measurement solvent polymeric membranes are used. As reference electrode a $\mathrm{pH}-\mathrm{ISFET}$ with a retarded $\mathrm{pH}$-response was developed thereby creating the possibility to operate this ISFET as an ion-insensitive FET over short periods of time (pseudo-REFET). The multi-ion sensor consisting of the pH-ISFET, $\mathrm{pK}$-ISFET and PCa-ISFET together with the pseudo-REFET shows a high precision in combination with a high-sample frequency. 
The cell, with an internal volume as small as $12 \mu$, is evaluated in connection with a FlAstaro now injection analysis system. The systern's performance is illustrated in Fig. 11. Into the carrier streas respectively samples of different pll, diffferent pK and different pCa were injected. Subsequently, a sample was measured in which the concentration of all three species was changed. As can bee seen from the width of the peaks, the response is very fast and up to at least 200 samples per hour can be analyzed. The small pll-peaks at the injection of the higher calcium ion concentration are caused by an actual decrease in pll and not by interference, and thus it can be concluded that the cross-talk between the sensors is negligible.

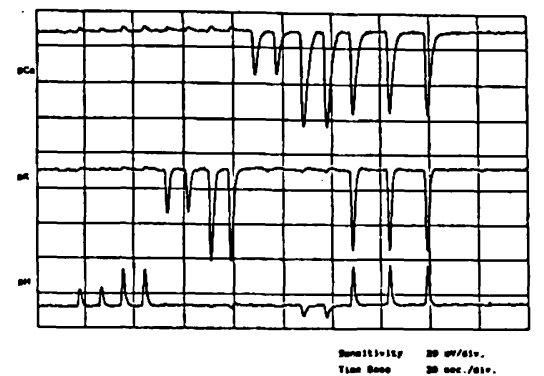

[3]

Fig. 11: Typical output registration of the flow-through cell in connection with an FA system

\subsection{Dlanar elucose sensor}

An amperometric glucose sensor (dimensions: $0.8 \mathrm{~mm}$ by $3 \mathrm{~mm}$ ) consisting of a planar three-electrode cell, an enzyme membrane and an outer polyurethane membrane, has been developed in our laboratory $[16,17]$.

The sensor is realized using thin-film deposition and lift-off techniques and consists of two Pt electrodes (working and counter) and an $\mathrm{Ag} / \mathrm{AgCl}$ reference electrode. It is coupled with an immobilized glucose oxidase membrane and the amount of $\mathrm{H}_{2} \mathrm{O}_{2}$ produced is measured at $0.7 \mathrm{~V}$. In order to render the sensor response less dependent of the dissolved oxygen concentration a polyurethane membrane is applied by dip-coating. The sensor response, measured at $37^{\circ} \mathrm{C}$ in a Ringer solution (pll 7.5) saturated with $5 \% \mathrm{O}_{2}$. is linear up to $40 \mathrm{mM}$ glucose with a sensitivity of 1.8 $\pm 0.2 \mathrm{nA} / \mathrm{mM}$. The operational lifetime, defined is the period during which the sensitivity remains constant, is approximately one week. The storage lifetime is more than one month. Recently the lift-off technique has been used for patteming enzymatic membranes on wafer level [18]. Sensors with on wafer deposited enzymatic membranes (without the polyurethane membrane) show a linear response to $1.5 \mathrm{mM}$ glucose and respond to about $6 \mathrm{mM}$ with a good uniformity amongst the sensors.

\section{Conclusions}

Many classical systems for medical diagnosis can be miniaturized effectively by the introduction of silicon integrated sensors. Moreover, the performance of the sensors is often better than that of the classical devices, and even new possibilities are created. This has been shown in this paper by examples of sensors that were mounted in catheters for invasive use in the human body. Also with senson for ex vivo use, such as the multi-ion sensing flow through cell, the small dimension are of great advantage to minimize the sample volume.

\section{Acknowiedrements}

The author would like to thank his coworken of the Microelectronic: Sensor Technology Group of the Institute of Microtechnology of the University of Neuchbiel as well as his colleagues of the Swiss Center for Electronice and Microtechnology Inc. (CSEM) for the many discussions, designs, skillful fabrication and chancterization of the sensors discussed in this paper. 

amperometric enzyme-hased slucuse microelectrode Senion and Actuaton de(1989) 157.105.

(18) S. Germer M Kouck tha, N.F. do Rooij. A planer glucnos enryme electrado. Sensurs and Actuators 22 81989) 537.540. 